\title{
Anleitung zur Benutzung des Hyperventilationstestes in der pneumologischen Praxis
}

D. Köhler

\author{
Manual for the Application of Hyperventilation Testing in Outpatient \\ Pulmonary Care
}

Ein relativ großer Teil der Patienten in der pneumologischen Praxis gibt anamnestisch Zustände von Luftnot an, ohne dass diese zum Vorstellungszeitpunkt vorhanden ist. Daher ist es eine wesentliche Aufgabe des Arztes, durch entsprechende Tests die Ursache der Dyspnoe herauszufinden. Dabei gehört neben dem Nachweis der normalen Lungenfunktion, der bronchialen Hyperreagibilitätstestung und den Belastungsblutgasen, der Hyperventilationstest ganz entscheidend dazu. Natürlich darf nicht vergessen werden, dass eine akute Hyperventilation mit zahlreichen Krankheitsbildern, vom Migräneanfall bis zur Lungenembolie einhergehen kann $[5,16]$.

Der Hyperventilationstest wird seit ca. 50 Jahren durchgeführt [11], wobei die Literatur gerade aus pneumologischer Sicht spärlich, aber zur Abklärung von unklarer Dyspnoe relativ eindeutig ist $[2-4,13]$. Für die Detektion von Panikattacken ist er relativ wenig hilfreich, da es häufig Panikattacken ohne Hyperventilation gibt $[1,6,9,10,14]$. Bei positivem Testausfall ist aber die Aussage hilfreich, da dieser Test kaum falsch-positive Reaktionen zeigt $[8,12]$. Manche Patienten zeigen eine isolierte, belastungsinduzierte Hyperventilation als alleinige Ursache der Dyspnoe [7]. Diese werden aber gut in den Belastungsgasen und noch besser in der Ergospirometrie erkannt.

Patienten, die einen positiven Testausfall haben, zeigen mit der willkürlich induzierten Hyperventilation oft Symptome, die denen zum Zeitpunkt der Dyspnoe entsprechen. Manche Patienten mit Asthma können aber auch zusätzlich eine Hyperventilation haben [15]. Anhand des Testes können diese Patienten oft genau sagen, ob die Luftnot jetzt durch Hyperventilation oder durch einen Asthmaanfall ausgelöst wurde. In Grenzfällen hilft natürlich die Mitgabe eines Peak flows, die zwischen beiden Zuständen klar separiert.

Der Test ist vergleichsweise einfach durchzuführen, wobei er aber immer unter Beobachtung stattfinden muss. Manche Patienten reagieren mit Kollapszuständen, so dass man den Test auf einem Stuhl mit hoher Lehne durchführen sollte, damit der Patient nicht umkippt.

In der Literatur wird meistens eine Hyperventilationszeit von 3 Minuten angegeben. Nach unserer Erfahrung reichen aber $2 \mathrm{Mi}$ nuten aktiver heftiger Hyperventilation aus. Wichtig ist die Blutgasmessung vorher, um eine bereits vorhandene Hyperventilation zu erkennen. Nach unseren Erfahrungen sollte der $\mathrm{pCO}_{2}$ vor dem Test über $32 \mathrm{~mm} \mathrm{Hg}$ liegen. Nach Beendigung der Hyperventilation sollte der $\mathrm{pCO}_{2}$ unter $25 \mathrm{~mm} \mathrm{Hg}$ fallen. Danach kreuzt die Praxismitarbeiterin die Symptome an, die sie beobachtet bzw. über die der Patient berichtet. Entscheidend ist die anschließende Frage, ob ähnliche Empfindungen schon früher aufgetreten sind, insbesondere zu dem Zeitpunkt an dem der Patient seine Luftnot verspürt hat, die ihn jetzt zum Arzt führt. Dieses wird notiert werden. Ggf. kann dann der Arzt hier noch einmal bei der Besprechung nachfassen.

Positiv ist der Test, wenn atypische Reaktionen auftreten. Schwindel und Kollapsneigung infolge Blutdruckabfall durch Hyperventilation haben auch Gesunde. Diese können aber in der Regel klar angeben, dass sie ähnliche Symptome nicht hatten, als die anamnestisch angegebene Luftnot auftrat.

Alternativ ist es auch möglich, das $\mathrm{CO}_{2}$ in der Exspirationsluft zu messen, wenn man über ein entsprechendes Gasanalysegerät 
Untersuchungsdatum:

Patient:

\section{Fragestellung:}

Blutgase vor:

$\left(\mathrm{PaCO}_{2}>32 \mathrm{mmHg}\right)$

Blutgase nach:

$\left(\mathrm{PaCO}_{2}\right.$ sollte $\left.<25 \mathrm{mmHg}\right)$

Hyperventilationszeit:

min.

(ca. 2min, Sitzen im Stuhl mit hoher Rückenlehne falls Kollapsneigung)

\section{Symptome:}

\begin{tabular}{|c|c|c|}
\hline Engegefühl in der Brust & Schwindel/ Schwarzwerden & \\
\hline Luftnot & Schweißausbruch & \\
\hline Schmerz in der Brust & Kalte feuchte Hände/ Füße & \\
\hline Trockengefühl des Mundes & Weiße Hände/ Blässe & \\
\hline Klos im Hals & Herzklopfen & \\
\hline Kribbeln Hände/ Finger/ Füße & Blutdruckabfall & \\
\hline Pfötchenstellung-Hände / Versteifungen & Angst & \\
\hline Muskelzittern & Weinen & \\
\hline Kopfschmerz/Kopfdruck & Sonstiges & \\
\hline Leeregefühl im Kopf & Keine Symptome & \\
\hline
\end{tabular}

Befund:

\section{Unterschrift}

verfügt. Dieses dürfte jedoch routinemäßig nicht verfügbar sein. Beiliegende Abb. 1 stellt das Beispiel einer Dokumentation dar, wie sie bei uns im Krankenhaus verwendet wird.

Wichtig ist es, den Test an unterschiedlichen Patientengruppen häufiger durchzuführen, so dass man eigene Erfahrungen über die Validität bekommt.

Eine medikamentöse Therapie der Hyperventilation ist meistens nicht nötig. Es reicht, die Patienten darauf hinzuweisen, dass dieser Zustand harmlos ist und sich selbst begrenzt. Damit sind die meisten Patienten beruhigt. Nur in schweren Fällen sollte man auf Sedativa oder Betablocker zurückgreifen. Sportliche Aktivität mit raschem Lastwechsel zum Training des Atmungszentrums hilft ebenfalls in manchen Fällen. Patienten mit positivem Hyperventilationstest zeigen übrigens häufiger auch andere vegetative Dysregulationen wie Palpitationen, Schweißausbrüche oder Dermographismus. Sie entsprechen wahrscheinlich einer Dysre- gulation des autonomen Nervensystems, die wohl angeboren sein dürfte. Psychotherapeutische Maßnahmen helfen nicht.

\section{Literatur}

${ }^{1}$ Bass C, Chambers JB, Kiff P et al. Panic anxiety and hyperventilation in patients with chest pain: a controlled study. Q J Med 1988; 69: 949-959

${ }^{2}$ Burton CD. Hyperventilation in patients with recurrent functional symptoms. Br J Gen Pract 1993; 43: 422 - 425

${ }^{3}$ Compernolle T, Hoogduin K, Joele L. Diagnosis and treatment of the hyperventilation syndrome. Psychosomatics 1979; 20: 612-625

${ }^{4}$ DePaso WJ, Winterbauer RH, Lusk JA et al. Chronic dyspnea unexplained by history, physical examination, chest roentgenogram, and spirometry. Analysis of a seven-year experience. Chest 1991; 100: $1293-1299$

${ }^{5}$ Folgering $\mathrm{H}$. The pathophysiology of hyperventilation syndrome. Monaldi Arch Chest Dis 1999; 54: 365-372 Review

${ }^{6}$ Garssen B, Buikhuisen M, Dyck R van. Hyperventilation and panic attacks. Am J Psychiatry 1996; 153: 513-518 
${ }^{7}$ Hammo AH, Weinberger MM. Exercise-induced hyperventilation: a pseudoasthma syndrome. Ann Allergy Asthma Immunol 1999; 82: $574-578$

${ }^{8}$ Han JN, Schepers R, Stegen K et al. Psychosomatic symptoms and breathing pattern. J Psychosom Res 2000; 49: 319-333

${ }^{9}$ Hornsveld HK, Garssen B, Dop MJ et al. Double-blind placebo-controlled study of the hyperventilation provocation test and the validity of the hyperventilation syndrome. Lancet 1996; 20; 348: 154-158

${ }^{10}$ Howell JB. The hyperventilation syndrome: a syndrome under threat? Thorax 1997; 52 Suppl 3: S30-34 Review

${ }^{11}$ Lewis BI. Hyperventilation syndrome. A clinical and physiological evaluation. Calf Med 1959; 21: 259-271

12 Lowhagen O, Arvidsson M, Bjarneman P et al. Exercise-induced respiratory symptoms are not always asthma. Respir Med 1999; 93: $734-738$

${ }^{13}$ Malmberg LP, Tamminen K, Sovijarvi AR. Orthostatic increase of respiratory gas exchange in hyperventilation syndrome. Thorax 2000; 55 : 295-301

${ }^{14}$ Nardi AE, Valenca AM, Nascimento I et al. Hyperventilation challenge test in panic disorder and depression with panic attacks. Psychiatry Res 2001; 15; 105: 57-65

${ }^{15}$ Ringsberg KC, Akerlind I. Presence of hyperventilation in patients with asthma-like symptoms but negative asthma test responses: provocation with voluntary hyperventilation and mental stress. J Allergy Clin Immunol 1999; 103: 601-608

${ }^{16}$ Saisch SG, Wessely S, Gardner WN. Patients with acute hyperventilation presenting to an inner-city emergency department. Chest 1996; 110: $952-957$

\section{Erratum}

\section{Müller, K.-M. Pleuramesotheliom - Pathologie und Pathoge- nese. Pneumologie 2004; 58: 670-679.}

Der englische Titel dieser Arbeit wurde falsch abgedruckt. Er lautet richtig: Pleuromesothelioma - Pathology and Pathogenesis.

Der Verlag bittet dieses Versehen zu entschuldigen. 